\title{
Diffusion-stress relations in polymer mixtures
}

\author{
C. F. Curtiss \\ Theoretical Chemistry Institute, Department of Chemistry, University of Wisconsin-Madison, Madison, \\ Wisconsin 53706
}

\section{R. Byron Bird}

Chemical Engineering Department and Rheology Research Center, University of Wisconsin-Madison, Madison, Wisconsin 53706

(Received 14 June 1999; accepted 15 September 1999)

\begin{abstract}
A new derivation of the mass-flux equations for multicomponent diffusion in polymeric liquids is given. A precursor of the Fokker-Planck equation for a single polymer molecule is used as the starting point for the development. From this equation we derive the equation of motion for one polymer species. Then it is shown what assumptions may be made for the self-correlation tensors in order to obtain relations of the form of the Maxwell-Stefan equations for multicomponent diffusion. These results, valid for any bead-spring model, interrelate the mass-flux vectors and the stress tensor. Finally, the results are compared with the Chapman-Enskog solution of the Boltzmann equation for the limiting case of an ideal gas mixture of rigid spheres (for which no assumptions need to be made for the self-correlation tensors). (c) 1999 American Institute of Physics.
\end{abstract}

[S0021-9606(99)51546-8]

\section{INTRODUCTION}

In the past several years we have published four papers ${ }^{1-4}$ dealing, at least in part, with the expression for the mass flux in polymeric liquid mixtures. In each of these we have made some approximations, and consequently slightly different results emerge from the four different theoretical treatments.

In the first paper $^{1}$ we developed a formal kinetic theory for polymeric liquid mixtures, starting with a general equation of change in the phase space of the entire liquid mixture [Ref. 1, Eq. (3.7)]. The polymers are modeled as structures of arbitrarily connected beads and springs. When, for such molecules, the general equation of change is specialized to the equation of change for the mass of one species $\alpha$ we found the mass flux in the Fick form [Ref. 1, Eq. (B.7)]

$$
\mathbf{j}_{\alpha} \frac{\zeta}{m}=-\left[\boldsymbol{\nabla} \cdot\left(\boldsymbol{\pi}_{\alpha}^{(k)}+\boldsymbol{\pi}_{\alpha}^{(\phi)}+\boldsymbol{\pi}_{\alpha}^{(e)}\right)\right]+\mathbf{G}_{\alpha}
$$

Here $\mathbf{j}_{\alpha}$ is the mass flux of species $\alpha$, and $\zeta$ and $m$ are the friction coefficient and mass of a bead, all beads for all species taken to be identical. In addition, $\mathbf{G}_{\alpha}$ is the external force per unit volume acting on species $\alpha$, and $\boldsymbol{\pi}_{\alpha}^{(k)}, \boldsymbol{\pi}_{\alpha}^{(\phi)}$, and $\boldsymbol{\pi}_{\alpha}^{(e)}$ are the kinetic, intramolecular, and external force contributions to the partial stress tensor for species $\alpha$. The intermolecular force contribution $\boldsymbol{\pi}_{\alpha}^{(d)}$ does not appear because we made the "short range force assumption," which states that the force between beads on two different chains is zero, unless the beads coincide. Three additional assumptions were made: neglect of acceleration terms, scalar friction coefficients, and all beads on all molecules with the same mass and the same friction coefficients. Equation (1.1) agrees with the continuum mechanics result given by Ottinger, ${ }^{5}$ and with the result of Beris and Mavrantzas ${ }^{6}$ for Rouse chains. Unfortunately, this result can apply only to a solute in a dilute solution; when Eq. (1.1) is summed over all species $\alpha$, the left-hand side vanishes, whereas the right-hand side does not.

The latter deficiency was remedied in the second paper, ${ }^{2}$ where the starting point was also the general equation of change for the multicomponent mixture. Here the sole approximation is contained in an ansatz for the force on a bead of species $\alpha$ by all the surrounding molecules [Ref. 2, Eq. (22)]. Embedded in the ansatz is an idea similar to the "short range force assumption.' The resulting diffusion flux equations are then of the Maxwell-Stefan form

$$
\begin{aligned}
\sum_{\beta} & \frac{Z_{\alpha \beta} \rho_{\alpha} \rho_{\beta}}{\rho}\left(\frac{\mathbf{j}_{\alpha}}{\rho_{\alpha}}-\frac{\mathbf{j}_{\beta}}{\rho_{\beta}}\right) \\
& =-\left[\boldsymbol{\nabla} \cdot\left(\boldsymbol{\pi}_{\alpha}^{(k)}+\boldsymbol{\pi}_{\alpha}^{(\phi)}+\boldsymbol{\pi}_{\alpha}^{(e)}\right)\right]+\mathbf{G}_{\alpha}+\frac{\rho_{\alpha}}{\rho}\left[\left[\boldsymbol { \nabla } \cdot \left(\boldsymbol{\pi}^{(k)}\right.\right.\right. \\
& \left.\left.\left.+\boldsymbol{\pi}^{(\phi)}+\boldsymbol{\pi}^{(e)}\right)\right]-\mathbf{G}\right]-\left[\mathbf{j}_{\alpha(1)}+\mathbf{j}_{\alpha}(\boldsymbol{\nabla} \cdot \mathbf{v})+\mathbf{j}_{\alpha} \cdot \dot{\gamma}+\mathbf{j}_{\alpha} \cdot \boldsymbol{\omega}\right] .
\end{aligned}
$$

Here $Z_{\alpha \beta}$ is a "friction coefficient," with units of $t^{-1}$, the $\boldsymbol{\pi}^{(\cdots)}$ are contributions to the total stress tensor, and $\mathbf{G}$ is the total external force per unit volume. In the last term on the right-hand side, $\mathbf{j}_{\alpha(1)}=\left(D \mathbf{j}_{\alpha} / D t\right)-\left[\mathbf{j}_{\alpha} \cdot \nabla \mathbf{v}\right]$ is a convected time derivative, and $\boldsymbol{\gamma}=\nabla \mathbf{v}+(\nabla \mathbf{v})^{\dagger}$ and $\boldsymbol{\omega}=\nabla \mathbf{v}-(\nabla \mathbf{v})^{\dagger}$ are the rate of deformation tensor and the vorticity tensor, respectively. Recently Johnson, ${ }^{7}$ using only the balance equations for mass, linear momentum, and angular momentum, along with empirical constitutive equations for the diffusive forces, obtained Eq. (1.2), but with $\boldsymbol{\pi}_{\alpha}^{(d)}$ and $\boldsymbol{\pi}^{(d)}$ included in the stress tensor terms, as well as a thermal diffusion term. No use was made of the energy or entropy balances.

In the third paper $^{3}$ we showed how to get a FokkerPlanck equation for the one-molecule distribution function and how to solve it by a perturbation procedure. Then, in 
Appendix $C$, Part 3, we used this solution to evaluate the formal expression for $\mathbf{j}_{\alpha}$ given in Eq. (6.10) of Table 2 of Ref. 1 When external forces are left out of consideration, this leads once again to equations of the Maxwell-Stefan form

$$
\begin{aligned}
& \sum_{\beta} \frac{Z_{\alpha \beta} \rho_{\alpha} \rho_{\beta}}{\rho}\left(\frac{\mathbf{j}_{\alpha}}{\rho_{\alpha}}-\frac{\mathbf{j}_{\beta}}{\rho_{\beta}}\right) \\
& \quad=-\left[\nabla \cdot\left(\boldsymbol{\pi}_{\alpha}^{(k)}+\boldsymbol{\pi}_{\alpha}^{(\phi)}\right)\right]+\frac{\rho_{\alpha}}{\rho}\left[\nabla \cdot\left(\boldsymbol{\pi}^{(k)}+\boldsymbol{\pi}^{(\phi)}\right)\right] .
\end{aligned}
$$

The last term in Eq. (1.2) does not appear here, since it is presumably 'of higher order' in the perturbation parameter $\varepsilon$ introduced in the derivation of the Fokker-Planck equation [Eq. (7.14) of Ref. 3]. In the derivation three assumptions are made, and in solving it a fourth assumption is introduced. In "Assumption 1" we argued that the term containing the pair distribution function is small and can be neglected, and as a result the terms containing $\boldsymbol{\pi}_{\alpha}^{(d)}$ and $\boldsymbol{\pi}^{(d)}$ do not appear. If, on the other hand, the "Assumption 1", is not used, the development can still be carried through, and then a good approximation to the additional terms does appear on the right-hand side.

In developing the Maxwell-Stefan form of the mass-flux vectors, we should be certain that our results are consistent with the analogous equations in the linear limit obtained from the thermodynamics of irreversible processes. This led to the fourth paper, ${ }^{4}$ which investigated various aspects of multicomponent diffusion, mass fluxes, and driving forces. The results for the mass fluxes are given in the MaxwellStefan form and are

$$
\begin{aligned}
c R T \sum_{\beta} \widetilde{C}_{\alpha \beta}\left(\frac{\mathbf{j}_{\alpha}}{\rho_{\alpha}}-\frac{\mathbf{j}_{\beta}}{\rho_{\beta}}\right) \\
=-\nabla p_{\alpha}+\rho_{\alpha} \mathbf{g}_{\alpha}+\frac{\rho_{\alpha}}{\rho}\left(\nabla p-\sum_{\beta} \rho_{\beta} \mathbf{g}_{\beta}\right) \\
\quad+c R T \sum_{\beta} \widetilde{C}_{\alpha \beta}\left(\frac{D_{\alpha}^{T}}{\rho_{\alpha}}-\frac{D_{\beta}^{T}}{\rho_{\beta}}\right)(\nabla \ln T)
\end{aligned}
$$

in which $\nabla p_{\alpha}=c_{\alpha} R T \nabla \ln a_{\alpha}+c_{\alpha}\left(\bar{H}_{\alpha}-\bar{H}_{\alpha}^{0}\right) \nabla \ln T$. Here $c_{\alpha}$ is the molar concentration of species $\alpha, c$ is the total molar density, the $\widetilde{C}_{\alpha \beta}$ are the (symmetric) "multicomponent inverse diffusivities," $a_{\alpha}$ is the activity of species $\alpha$, and $\bar{H}_{\alpha}$ is the partial molar enthalpy (and the superscript ' 0 '" indicates the standard state value). The symbol $\mathbf{g}_{\alpha}$ is the external force per unit mass acting on species $\alpha$. The last term on the righthand side of Eq. (1.4) is the contribution from thermal diffusion.

In this paper we take a precursor to the Fokker-Planck equation [Eqs. (7.1) to (7.5) of Ref. 3] as the starting point, and write it in the form of a multidimensional Boltzmann equation in the full phase space of a single molecule. On the left-hand side we introduce the known zeroth-order (equilibrium) solution, and on the right-hand side the (unknown) first-order solution, thus obtaining the analog of the linearized Boltzmann equation. Then following the procedure of Sec. 7 of Ref. 1, we multiply this equation by $\delta\left(\mathbf{r}_{\nu}^{\alpha}-\mathbf{r}\right) \mathbf{p}_{\nu}^{\alpha}$, sum on $\nu$, and integrate over the one-molecule phase space to develop an equation of motion for species $\alpha$. Working "at the lowest order' in the parameter $\varepsilon$ introduced in Ref. 3, we get the equation of motion for species $\alpha$, which, after several assumptions for the self-correlation tensor, gives the following diffusion-stress relation:

$$
\begin{aligned}
\sum_{\beta} & \frac{\overline{\mathbf{Z}}_{\alpha \beta} \rho_{\alpha} \rho_{\beta}}{m_{0}^{\alpha} m_{0}^{\beta} k T} \cdot\left(\frac{\mathbf{j}_{\alpha}}{\boldsymbol{\rho}_{\alpha}}-\frac{\mathbf{j}_{\beta}}{\rho_{\beta}}\right) \\
\quad & =-\left[\boldsymbol{\nabla} \cdot \boldsymbol{\pi}_{\alpha}-\mathbf{G}_{\alpha}\right]+\frac{\rho_{\alpha}}{\rho}[\boldsymbol{\nabla} \cdot \boldsymbol{\pi}-\mathbf{G}]
\end{aligned}
$$

in which $\boldsymbol{\pi}_{\alpha}=\boldsymbol{\pi}_{\alpha}^{(k)}+\boldsymbol{\pi}_{\alpha}^{(\phi)}+\boldsymbol{\pi}_{\alpha}^{(e)}+\boldsymbol{\pi}_{\alpha}^{(d)}$ is the partial stress tensor for species $\alpha$, and $\boldsymbol{\pi}=\boldsymbol{\pi}^{(k)}+\boldsymbol{\pi}^{(\phi)}+\boldsymbol{\pi}^{(e)}+\boldsymbol{\pi}^{(d)}$ is the total stress tensor. The symbols $m_{0}^{\alpha}$ and $m_{0}^{\beta}$ are the bead masses of molecules $\alpha$ and $\beta$, with all bead masses for all the molecules of a given type taken to be the same. This expression is missing the last term in Eq. (1.2) and the last term in Eq. (1.4), both of which are believed to be small.

In deriving Eq. (1.5) we have made as few assumptions as possible about the correlation tensors. The second-order symmetric friction tensors $\overline{\mathbf{Z}}_{\alpha \beta}$-closely related to the correlation tensors-have units of $m^{2} l^{5} / t^{3}$. Note that Eq. (1.5) differs in form from the customary Maxwell-Stefan equations in that the mass fluxes are operated on by tensors; the resulting lack of alignment of the fluxes with the driving forces may be a characteristic of systems composed of molecules with complex structure.

\section{LINEARIZATION OF THE TIME-EVOLUTION EQUATION OF THE SINGLET DISTRIBUTION FUNCTION}

We model the polymer molecules as collections of "beads" joined together by "springs" in an arbitrary arrangement and numbered arbitrarily. The symbols $m_{\nu}^{\alpha}, \mathbf{r}_{\nu}^{\alpha}$, and $\mathbf{p}_{\nu}^{\alpha}$ stand for the mass, position vector, and momentum of bead $\nu$ of molecule of species $\alpha$. The quantity $\mathbf{F}_{\nu}^{\alpha}$ is the total force on bead $\nu$ of species $\alpha$ exerted by all the other beads and also by external forces.

In Ref. 3, Eqs. (7.1)-(7.3), we developed a set of equations for the time-averaged single-molecule distribution function $\bar{f}_{\alpha}$ (in this paper we omit the time-average overbar). Here we rewrite these equations in the following form:

$$
\begin{aligned}
\frac{\partial}{\partial t} f_{\alpha} & +\sum_{\nu}\left(\frac{1}{m_{\nu}^{\alpha}} \mathbf{p}_{\nu}^{\alpha} \cdot \frac{\partial}{\partial \mathbf{r}_{\nu}^{\alpha}} f_{\alpha}\right)+\sum_{\nu}\left(\mathbf{F}_{\nu}^{\alpha} \cdot \frac{\partial}{\partial \mathbf{p}_{\nu}^{\alpha}} f_{\alpha}\right) \\
= & -\sum_{\nu}\left(\frac{\partial}{\partial \mathbf{p}_{\nu}^{\alpha}} \cdot\left(\mathcal{F}_{\nu}^{(b) \alpha}+\mathcal{F}_{\nu}^{(h) \alpha}\right) f_{\alpha}\right)
\end{aligned}
$$

in which $\mathcal{F}_{\nu}^{(b) \alpha}$ and $\mathcal{F}_{\nu}^{(h) \alpha}$ are the "Brownian" and "hydrodynamic" forces. Explicit expressions for these quantities appear in Eqs. (7.4) and (7.5) of Ref. 3 in terms of the derivatives of the distribution functions and the averaged correlation tensors defined there. Furthermore the force $\mathbf{F}_{\nu}^{\alpha}$ can be written as 


$$
\mathbf{F}_{\nu}^{\alpha}=\mathbf{F}_{\nu}^{(\phi) \alpha}\left(\mathbf{r}^{\alpha}\right)+\mathbf{F}_{\nu}^{(d) \alpha}\left(\mathbf{r}^{\alpha}\right)+\mathbf{F}_{\nu}^{(e) \alpha}\left(\mathbf{r}^{\alpha}\right)
$$

that is, a sum of contributions to the force on bead $\nu$ of molecule $\alpha$ resulting from intramolecular forces ("springs"), intermolecular forces (represented, for example, by a 'Lennard-Jones potential') and external forces (gravity or electrical forces). The term $\mathbf{F}_{\nu}^{(d) \alpha}$ is given as one of the terms in Eq. (7.3) of Ref. 3, which, when corrected for misprints, is

$$
\mathbf{F}_{\nu}^{(d) \alpha}=\sum_{\beta} \sum_{\mu} \int g_{\alpha \beta, 0}\left(\mathbf{r}^{\alpha}, \mathbf{r}^{\beta}\right) f_{\beta}\left(x^{\beta}, t\right) \mathbf{F}_{\nu \mu}^{(d) \alpha \beta} d x^{\beta}
$$

in which $g_{\alpha \beta, 0}$ is the local equilibrium radial distribution function defined by Eq. (6.5) of Ref. 3. Note that from the definition of $f_{h 0}^{\alpha \beta}$ [see the discussion after Eq. (5.5) of Ref. 3] $g_{\alpha \beta, 0}$ is a function in the configuration space of the two molecules and is independent of the momentum coordinates. By including this contribution to $\mathbf{F}_{\nu}^{\alpha}$, we avoid making Assumption 1 on p. 9907 of Ref. 3.

Our development ${ }^{3}$ of Eq. (2.1) was inspired by Kirkwood's use of time-averaging ${ }^{8}$ in the derivation of FokkerPlanck equations restricted to mixtures of monatomic molecules. Equation (2.1) is thus essentially a generalization of Kirkwood's Eq. (59) to polymeric mixtures, although the details of the developments are different.

The left-hand side of Eq. (2.1) is a "substantial derivative', in the phase space, $\left\{x^{\alpha}\right\}=\left\{\mathbf{r}^{\alpha}, \mathbf{p}^{\alpha}\right\}$, and is designated by $\mathrm{D} / \mathrm{D} t$. The right-hand side can be designated by $J_{\alpha}$. Then Eq. (2.1) can be written as

$$
\frac{\mathbb{D} f_{\alpha}}{\mathrm{D} t}=J_{\alpha}
$$

This can be regarded as an analog to the simple Boltzmann equation [Eq. (7.1-25) of Ref. 9]. Note that the true forces (intermolecular, intramolecular, and external) are on the lefthand side in the phase-space substantial derivative operator $\mathrm{D} / \mathrm{D} t$, and the chaotic forces (Brownian and hydrodynamic) are in $J_{\alpha}$, which is the analog of the Boltzmann collision integral in dilute gas kinetic theory.

The explicit expressions for the Brownian and hydrodynamic forces are given in Ref. 3, Eqs. (7.4) and (7.5). When we make use of "Assumption 2', and neglect all correlations except self-correlations, the expression for $J_{\alpha}$ becomes

$$
\begin{aligned}
J_{\alpha}= & -\sum_{\nu \beta \mu} \frac{\partial}{\partial \mathbf{p}_{\nu}^{\alpha}} \cdot \int f_{\alpha} f_{\beta} \mathbf{E}_{\nu \mu}^{\alpha \beta} \cdot\left(\frac{\partial}{\partial \mathbf{p}_{\nu}^{\alpha}} \ln \frac{f_{\alpha}}{f_{\alpha 0}}\right) d x^{\beta} \\
& -\sum_{\nu \beta \mu} \frac{\partial}{\partial \mathbf{p}_{\nu}^{\alpha}} \cdot \int f_{\alpha} f_{\beta} \mathbf{E}_{\mu \nu}^{\beta \alpha} \cdot\left(\frac{\partial}{\partial \mathbf{p}_{\mu}^{\beta}} \ln \frac{f_{\beta}}{f_{\beta 0}}\right) d x^{\beta} .
\end{aligned}
$$

The averaged self-correlation tensors are symmetric and also have the symmetry property that $\mathbf{E}_{\mu \nu}^{\beta \alpha}\left(x^{\beta}, x^{\alpha}, t\right)$ $=\mathbf{E}_{\nu \mu}^{\alpha \beta}\left(x^{\alpha}, x^{\beta}, t\right)$. The quantities $f_{\alpha 0}$ and $f_{\beta 0}$ are the momentum-equilibrated distribution functions given by Eq. (5.5) of Ref. 3:

$$
f_{\alpha 0}\left(x^{\alpha}, t\right)=\frac{\Psi_{\alpha}\left(\mathbf{r}^{\alpha}, t\right) \exp \left[-\sum_{\nu=1}^{N_{\alpha}}\left[\mathbf{p}_{\nu}^{\alpha}-m_{\nu}^{\alpha} \mathbf{v}\left(\mathbf{r}_{\nu}^{\alpha}, t\right)\right]^{2} / 2 m_{\nu}^{\alpha} k T\left(\mathbf{r}_{\nu}^{\alpha}, t\right)\right]}{\Pi_{\nu=1}^{3 N_{\alpha}}\left[2 \pi m_{\nu}^{\alpha} k T\left(\mathbf{r}_{\nu}^{\alpha}, t\right)\right]^{1 / 2}}=\frac{\Psi_{\alpha}\left(\mathbf{r}^{\alpha}, t\right) \exp \left(-P^{\alpha 2}\right)}{J_{\alpha} \pi^{3 N_{\alpha} / 2}}
$$

Here $\mathbf{v}\left(\mathbf{r}_{\nu}^{\alpha}, t\right)$ is the instantaneous mass-averaged velocity at bead $\mathbf{r}_{\nu}^{\alpha}$, and $T\left(\mathbf{r}_{\nu}^{\alpha}, t\right)$ is the corresponding instantaneous temperature at that same position. The symbol $\mathbf{P}^{\alpha}$ is defined by Eq. (9.1) of Ref. 3 and is a vector in the $3 N_{\alpha}$ momentum space, $J_{\alpha}$ is a normalization constant, and $\Psi_{\alpha}$ is a nonequilibrium distribution function in configuration space. Note that in the analogous statistical mechanical treatment of dilute gases and liquids of monatomic molecules, the analog of $\Psi_{\alpha}$ is a function in the three-dimensional physical space. For this reason the linearization discussed here leads, for dilute gases, to the "linear laws" of irreversible thermodynamics, whereas, for polymers, it leads to nonlinear expressions.

We now want to solve Eq. (2.4) [with $J_{\alpha}$ given by Eq. (2.5)] by means of perturbation theory and keep only the lowest-order terms in the parameter $\varepsilon$ defined in Ref. 3, p. 9903 , column 1, as the ratio of two time scales. Thus, since $f_{\alpha 0}$ is the distribution function in the limit that $\varepsilon \rightarrow 0$, we may write $f_{\alpha} / f_{\alpha 0}=1+O(\varepsilon)$, and also $\ln \left(f_{\alpha} / f_{\alpha 0}\right)=\left[\left(f_{\alpha} / f_{\alpha 0}\right)-1\right]$ $+O\left(\varepsilon^{2}\right)$. Then

$$
\begin{aligned}
\mathrm{J}_{\alpha}= & \sum_{\nu \beta \mu} \frac{\partial}{\partial \mathbf{p}_{\nu}^{\alpha}} \cdot \int f_{\alpha} f_{\beta} \mathbf{E}_{\nu \mu}^{\alpha \beta} \\
& \cdot\left[\frac{\partial}{\partial \mathbf{p}_{\nu}^{\alpha}}\left(\frac{f_{\alpha}}{f_{\alpha 0}}\right)-\frac{\partial}{\partial \mathbf{p}_{\mu}^{\beta}}\left(\frac{f_{\beta}}{f_{\beta 0}}\right)+O\left(\varepsilon^{2}\right)\right] d x^{\beta},
\end{aligned}
$$

where use has been made of the symmetry property of the $\mathbf{E}_{\nu \mu}^{\alpha \beta}$.

From our previous experience in solving the FokkerPlanck equation ${ }^{3}$ we know that the tensor Hermite polynomials are particularly useful for molecules with complex structure. Therefore we expand the solution in the form [see Ref. 3, Eq. (9.2)]:

$$
f_{\alpha}\left(x^{\alpha}, t\right)=e^{-P^{\alpha 2}} \sum_{n=0}^{\infty} \mathbf{h}_{\alpha}^{[n]}\left(\mathbf{P}^{\alpha}\right) \stackrel{n}{\odot} \mathbf{f}_{\alpha}^{[n]}\left(\mathbf{r}^{\alpha}, t\right)
$$

in which the superscript $[n]$ indicates an $n$th order tensor, the notation $\odot$ (called · in Ref. 3) denotes an $n$-fold " dot prod- 
uct' ' in $3 N_{\alpha}$-space, where $N_{\alpha}$ is the number of beads describing species $\alpha$. The expansion coefficients $\mathbf{f}_{\alpha}^{[n]}$ are given by

$$
\mathbf{f}_{\alpha}^{[n]}\left(\mathbf{r}^{\alpha}, t\right)=\int f_{\alpha}\left(x^{\alpha}, t\right) \mathbf{h}_{\alpha}^{[n]}\left(\mathbf{P}^{\alpha}\right) d \mathbf{P}^{\alpha}
$$

Then the ratio $f_{\alpha} / f_{\alpha 0}$ appearing in Eq. (2.7) is

$$
\frac{f_{\alpha}}{f_{\alpha 0}}=\sum_{n=0}^{\infty} \frac{\mathbf{h}_{\alpha}^{[n]} \bigodot^{n} \mathbf{f}_{\alpha}^{[n]}}{h_{\alpha}^{[0]} f_{\alpha}^{[0]}}=1+\sum_{n=1}^{\infty} \frac{\mathbf{h}_{\alpha}^{[n]} \odot^{n} \mathbf{f}_{\alpha}^{[n]}}{h_{\alpha}^{[0]} f_{\alpha}^{[0]}}
$$

in which $h_{\alpha}^{[0]}=1 / \pi^{3 N_{\alpha} / 4}$ [cf. Ref. 3, Eq. (A30)] and $f_{\alpha}^{[0]}$ $=\left(\Psi_{\alpha} / J_{\alpha} \pi^{3 N_{\alpha} / 4}\right)=\left(\Psi_{\alpha} / J_{\alpha}\right) h_{\alpha}^{[0]} \quad$ [cf. Ref. 3, Eq. (5.5)]. Then $\mathbb{J}_{\alpha}$ becomes

$$
\begin{aligned}
\mathbb{J}_{\alpha}= & \sum_{\nu \beta \mu} \sum_{n=1}^{\infty} \frac{\partial}{\partial \mathbf{p}_{\nu}^{\alpha}} \cdot \int f_{\alpha} f_{\beta} \mathbf{E}_{\nu \mu}^{\alpha \beta} \cdot\left[\frac{\partial}{\partial \mathbf{p}_{\nu}^{\alpha}} \frac{\mathbf{h}_{\alpha}^{[n]} \bigodot^{n} \mathbf{f}_{\alpha}^{[n]}}{h_{\alpha}^{[0]} f_{\alpha}^{[0]}}\right. \\
& \left.-\frac{\partial}{\partial \mathbf{p}_{\mu}^{\beta}} \frac{\mathbf{h}_{\beta}^{[n]} \odot_{\beta}^{n} \mathbf{f}_{\beta}^{[n]}}{h_{\beta}^{[0]} f_{\beta}^{[0]}}+O\left(\varepsilon^{2}\right)\right] d x^{\beta} .
\end{aligned}
$$

Now, again working at the lowest order in $\varepsilon$, the ratio $f_{\alpha} / f_{\alpha}^{[0]}$ appearing here is

$$
\frac{f_{\alpha}}{f_{\alpha}^{[0]}}=e^{-P^{\alpha 2}} \sum_{n=0}^{\infty} \mathbf{h}_{\alpha}^{[n]} \bigodot_{f_{\alpha}^{n}}^{\frac{\mathbf{f}_{\alpha}^{[n]}}{f_{\alpha}^{[0]}}}=e^{-P^{\alpha 2}} h_{\alpha}^{[0]}+O(\varepsilon)
$$

and

$$
\frac{f_{\alpha} f_{\beta}}{f_{\alpha}^{[0]}}=e^{-P^{\alpha 2}} h_{\alpha}^{[0]}\left(e^{-P^{\beta 2}} h_{\beta}^{[0]} f_{\beta}^{[0]}+O(\varepsilon)\right)+O(\varepsilon),
$$

so that

$$
\begin{aligned}
\mathbb{J}_{\alpha}= & \sum_{\nu \beta \mu} \sum_{n=1}^{\infty} h_{\alpha}^{[0]} h_{\beta}^{[0]} \frac{\partial}{\partial \mathbf{p}_{\nu}^{\alpha}} \cdot \int e^{-P^{\alpha 2}} e^{-P^{\beta 2}} \mathbf{E}_{\nu \mu}^{\alpha \beta} \\
& \cdot\left[\frac{\partial}{\partial \mathbf{p}_{\nu}^{\alpha}} \frac{\mathbf{h}_{\alpha}^{[n]}}{h_{\alpha}^{[0]}} \bigodot^{n} \mathbf{f}_{\alpha}^{[n]} f_{\beta}^{[0]}+O\left(\varepsilon^{2}\right)-\frac{\partial}{\partial \mathbf{p}_{\mu}^{\beta}} \frac{\mathbf{h}_{\beta}^{[n]} h_{\beta}^{n}}{h_{\beta}^{[0]}} \mathbf{f}_{\beta}^{[n]} f_{\alpha}^{[0]}\right. \\
& \left.+O\left(\varepsilon^{2}\right)\right] d x^{\beta} .
\end{aligned}
$$

From arguments similar to those in Ref. 3 [see, in particular Eqs. (7.9) and (9.10)] it follows that all the $\mathbf{E}_{\nu \mu}^{\alpha \beta}$ are $O(1 / \varepsilon)$. Thus if we drop all $O(\varepsilon)$ terms in $\mathrm{J}_{\alpha}$ and $\mathrm{D} f_{\alpha} / \mathrm{D} t$, we get to terms of zero order in $\varepsilon$

$$
\frac{\mathrm{D} f_{\alpha 0}}{\mathrm{D} t}=\mathbb{J}_{\alpha 0}
$$

which is the linearized time evolution equation for $f_{\alpha}\left(x^{\alpha}, t\right)$ analogous to the "linearized Boltzmann equation" in dilute gas kinetic theory [see Ref. 9, Eq. (7.3-22)].

\section{DERIVATION OF THE MAXWELL-STEFAN EQUATIONS FOR THE MASS FLUX}

In Ref. 2 we showed how to get the Maxwell-Stefan equations from the equation of motion for species $\alpha$ by specializing the general equation of change, which in turn comes from the Liouville equation for the entire fluid mixture. Here we perform a similar derivation, starting from the linearized equation in Eq. (2.15).

We multiply both sides of Eq. (2.15) by $\delta\left(\mathbf{r}_{\nu}^{\alpha}-\mathbf{r}\right) \mathbf{p}_{\nu}^{\alpha}$, sum on $\nu$, and integrate over all $x^{\alpha}$, thereby generating an equation of motion for species $\alpha$. Then we integrate the right-hand side $\left(\mathbb{J}_{\alpha 0}\right)$ by parts to get

$$
\begin{aligned}
& \sum_{\nu=1}^{N_{\alpha}} \int\left(\delta\left(\mathbf{r}_{\nu}^{\alpha}-\mathbf{r}\right) \mathbf{p}_{\nu}^{\alpha}\right)\left(\frac{\mathrm{D} f_{\alpha 0}}{\mathrm{D} t}\right) d x^{\alpha} \\
& =\sum_{\beta \mu \nu} h_{\alpha}^{[0]} h_{\beta}^{[0]} \sum_{n=1}^{\infty} \iint \delta\left(\mathbf{r}_{\nu}^{\alpha}-\mathbf{r}\right) e^{-P^{\alpha 2}} e^{-P^{\beta 2}} \mathbf{E}_{\nu \mu}^{\alpha \beta} \\
& \cdot\left[\frac{\partial}{\partial \mathbf{p}_{\mu}^{\beta}} \frac{\mathbf{h}_{\beta}^{[n]}}{h_{\beta}^{[0]}} \odot^{n} \mathbf{f}_{\beta}^{[n]} f_{\alpha}^{[0]}-\frac{\partial}{\partial \mathbf{p}_{\nu}^{\alpha}} \frac{\mathbf{h}_{\alpha}^{[n]}}{h_{\alpha}^{[0]}} \bigodot^{n} \mathbf{f}_{\alpha}^{[n]} f_{\beta}^{[0]}\right] d x^{\alpha} d x^{\beta} .
\end{aligned}
$$

This equation is analogous to Eq. (9.30) of Ref. 3, with the sides of the equations interchanged, except that here we have introduced $\mathbf{p}_{\nu}^{\alpha}$ rather than $\mathbf{h}_{\alpha \nu}^{[1]}$ and summed on $\nu$. Of course, there is a term missing from Eq. (9.30) because of "Assumption 1.',

In Ref. 3, Eq. (9.24), the equations for the $\mathbf{f}_{\alpha}^{[n]}$ are decoupled because of the assumptions made there. Here we have not made those assumptions and therefore the analogous set of equations would not be decoupled. In order to proceed we choose to consider only the $n=1$ term in Eq. (3.1) [which corresponds to the first equation in the set given by Eq. (9.24) of Ref. 3]. Because this is done, we do not get the thermal diffusion effect in our final result. We conjecture that inclusion of the term with $n=3$ would describe thermal diffusion. Note, however, that if we then assume that $\overline{\mathbf{E}}_{\nu \mu}^{\alpha \beta}$ in Eq. (3.13) is independent of momenta (i.e., if we assume that $\left[\left[\overline{\mathbf{E}}_{\nu \mu}^{\alpha \beta}\right]\right]_{0}=\overline{\mathbf{E}}_{\nu \mu}^{\alpha \beta}$, then the term with $n=3$ is zero.

From Ref. 3, Eqs. (A12), (A17), and (9.1), we get $\mathbf{h}_{\alpha}^{[1]}$ $=\sqrt{2} h_{\alpha}^{[0]} \mathbf{P}^{\alpha}$ (a vector in the chain space) and $\left(\partial / \partial \mathbf{p}_{\nu}^{\alpha}\right) \mathbf{h}_{\alpha \nu}^{[1]}$ $=\sqrt{1 / m_{\nu}^{\alpha} k T} h_{\alpha}^{[0]} \boldsymbol{\delta}$ (a second-order tensor in 3-space). Using these expressions in Eq. (3.1), we then get

$$
\begin{aligned}
\sum_{\nu=1}^{N_{\alpha}} \int & \left(\delta\left(\mathbf{r}_{\nu}^{\alpha}-\mathbf{r}\right) \mathbf{p}_{\nu}^{\alpha}\right)\left(\frac{\mathrm{D} f_{\alpha 0}}{\mathrm{D} t}\right) d x^{\alpha} \\
= & \sum_{\beta \mu \nu} h_{\alpha}^{[0]} h_{\beta}^{[0]} \iint \delta\left(\mathbf{r}_{\nu}^{\alpha}-\mathbf{r}\right) e^{-P^{\alpha 2}} e^{-P^{\beta 2}} \mathbf{E}_{\nu \mu}^{\alpha \beta} \\
& \cdot\left[\frac{1}{\sqrt{m_{\mu}^{\beta} k T_{\mu}^{\beta}}} \mathbf{f}_{\beta \mu}^{[1]} f_{\alpha}^{[0]}-\frac{1}{\sqrt{m_{\nu}^{\alpha} k T_{\nu}^{\alpha}}} \mathbf{f}_{\alpha \nu}^{[1]} f_{\beta}^{[0]}\right] d x^{\alpha} d x^{\beta}
\end{aligned}
$$

Now we proceed to develop the left-hand and right-hand sides of this equation. 


\section{A. Left-hand side of Eq. (3.2)}

First we write out $\mathbb{D} f_{\alpha 0} / \mathbb{D} t$ in full

$$
\begin{aligned}
\sum_{\nu=1}^{N_{\alpha}} \int & \left(\delta\left(\mathbf{r}_{\nu}^{\alpha}-\mathbf{r}\right) \mathbf{p}_{\nu}^{\alpha}\right)\left(\frac{\partial}{\partial t}+\sum_{\eta} \frac{\mathbf{p}_{\eta}^{\alpha}}{m_{\eta}^{\alpha}} \cdot \frac{\partial}{\partial \mathbf{r}_{\eta}^{\alpha}}+\sum_{\eta} \mathbf{F}_{\eta}^{\alpha} \cdot \frac{\partial}{\partial \mathbf{p}_{\eta}^{\alpha}}\right) \\
& \times\left(\frac{e^{-P^{\alpha 2}} \Psi_{\alpha}}{J_{\alpha} \pi^{3 N_{\alpha} / 2}}\right) d x^{\alpha} \\
= & \frac{h_{\alpha}^{[0] 2}}{J_{\alpha}} \sum_{\nu}\left[\frac{\partial}{\partial t} \iint \delta\left(\mathbf{r}_{\nu}^{\alpha}-\mathbf{r}\right) \mathbf{p}_{\nu}^{\alpha} e^{-P^{\alpha 2}} \Psi_{\alpha} d \mathbf{r}^{\alpha} d \mathbf{p}^{\alpha}\right. \\
& +\frac{1}{m_{\nu}^{\alpha}} \boldsymbol{\nabla} \cdot \iint \delta\left(\mathbf{r}_{\nu}^{\alpha}-\mathbf{r}\right) \mathbf{p}_{\nu}^{\alpha} \mathbf{p}_{\nu}^{\alpha} e^{-P^{\alpha 2}} \Psi_{\alpha} d \mathbf{r}^{\alpha} d \mathbf{p}^{\alpha} \\
& \left.-\iint \delta\left(\mathbf{r}_{\nu}^{\alpha}-\mathbf{r}\right) \mathbf{F}_{\nu}^{\alpha} e^{-P^{\alpha 2}} \Psi_{\alpha} d \mathbf{r}^{\alpha} d \mathbf{p}^{\alpha}\right] .
\end{aligned}
$$

To get the second and third terms we integrated by parts; in the second term we also made use of the antisymmetric property of the delta function. We now develop each of these three terms:

$$
\begin{aligned}
1 \mathrm{st} & =\left(\frac{\partial}{\partial t} \int \sum_{\nu} m_{\nu}^{\alpha} \delta\left(\mathbf{r}_{\nu}^{\alpha}-\mathbf{r}\right) \mathbf{v}\left(\mathbf{r}_{\nu}^{\alpha}, t\right) \Psi_{\alpha} d \mathbf{r}^{\alpha}\right) \\
& \times\left(\frac{h_{\alpha}^{[0] 2}}{J_{\alpha}} \int e^{-P^{\alpha 2}} d \mathbf{p}_{\nu}^{\alpha}\right) \\
= & \left(\frac{\partial}{\partial t} \mathbf{v}(\mathbf{r}, t) \sum_{\nu} m_{\nu}^{\alpha} \int \delta\left(\mathbf{r}_{\nu}^{\alpha}-\mathbf{r}\right) \Psi_{\alpha} d \mathbf{r}^{\alpha}\right) \\
= & \frac{\partial}{\partial t} \rho_{\alpha} \mathbf{v}(\mathbf{r}, t) .
\end{aligned}
$$

In going from the second to the third line we used Eq. (6.7) of Ref. 1.

$$
\begin{aligned}
2 \mathrm{nd}= & \sum_{\nu} \frac{h_{\alpha}^{[0] 2}}{J_{\alpha}} \frac{1}{m_{\nu}^{\alpha}} \boldsymbol{\nabla} \cdot \iint \delta\left(\mathbf{r}_{\nu}^{\alpha}-\mathbf{r}\right)\left[\left(\mathbf{p}_{\nu}^{\alpha}-m_{\nu}^{\alpha} \mathbf{v}\left(\mathbf{r}_{\nu}^{\alpha}, t\right)\right)\right. \\
& \times\left(\mathbf{p}_{\nu}^{\alpha}-m_{\nu}^{\alpha} \mathbf{v}\left(\mathbf{r}_{\nu}^{\alpha}, t\right)\right)+m_{\nu}^{\alpha} \mathbf{v}\left(\mathbf{r}_{\nu}^{\alpha}, t\right)\left(\mathbf{p}_{\nu}^{\alpha}-m_{\nu}^{\alpha} \mathbf{v}\left(\mathbf{r}_{\nu}^{\alpha}, t\right)\right) \\
& +m_{\nu}^{\alpha}\left(\mathbf{p}_{\nu}^{\alpha}-m_{\nu}^{\alpha} \mathbf{v}\left(\mathbf{r}_{\nu}^{\alpha}, t\right)\right) \mathbf{v}\left(\mathbf{r}_{\nu}^{\alpha}, t\right)+m_{\nu}^{\alpha 2} \mathbf{v}\left(\mathbf{r}_{\nu}^{\alpha}, t\right) \\
& \left.\times \mathbf{v}\left(\mathbf{r}_{\nu}^{\alpha}, t\right)\right] e^{-P^{\alpha 2}} \Psi_{\alpha} d \mathbf{r}^{\alpha} d \mathbf{p}_{\nu}^{\alpha} \\
= & \sum_{\nu} \frac{1}{m_{\nu}^{\alpha}} \boldsymbol{\nabla} \cdot\left[m_{\nu}^{\alpha} k T \boldsymbol{\delta} \int \delta\left(\mathbf{r}_{\nu}^{\alpha}-\mathbf{r}\right) \Psi_{\alpha} d \mathbf{r}^{\alpha}\right. \\
& \left.+m_{\nu}^{\alpha 2} \mathbf{v}(\mathbf{r}, t) \mathbf{v}(\mathbf{r}, t) \int \delta\left(\mathbf{r}_{\nu}^{\alpha}-\mathbf{r}\right) \Psi_{\alpha} d \mathbf{r}^{\alpha}\right] \\
= & {\left[\boldsymbol{\nabla} \cdot \boldsymbol{\pi}^{(k) \alpha}\right]+\left[\boldsymbol{\nabla} \cdot \rho_{\alpha} \mathbf{v v}\right] . }
\end{aligned}
$$

Here it is understood that $\boldsymbol{\pi}^{(k) \alpha}=\Sigma_{\nu}\left(\rho_{\nu}^{\alpha} / m_{\nu}^{\alpha}\right) k T \boldsymbol{\delta}$, that is, the expression calculated from Eq. (7.8) of Ref. 1, using the equilibrium momentum distribution function, as implied by the $f_{\alpha 0}$ on the left-hand side of Eq. (3.2).

$$
\begin{aligned}
3 \mathrm{rd} & =-\sum_{\nu} \int \delta\left(\mathbf{r}_{\nu}^{\alpha}-\mathbf{r}\right) \mathbf{F}_{\nu}^{\alpha} \Psi_{\alpha} d \mathbf{r}^{\alpha} \\
& =-\sum_{\nu} \int \delta\left(\mathbf{r}_{\nu}^{\alpha}-\mathbf{r}\right)\left(\mathbf{F}_{\nu}^{(\phi) \alpha}+\mathbf{F}_{\nu}^{(d) \alpha}+\mathbf{F}_{\nu}^{(e) \alpha}\right) \Psi_{\alpha} d \mathbf{r}^{\alpha} .
\end{aligned}
$$

We now consider the three terms in Eq. (3.6) separately.

(i) The first term, containing the intramolecular force $\mathbf{F}_{\nu}^{(\phi) \alpha}$, can be developed analogously to the procedure in going from Eq. (7.14) to Eq. (7.15) of Ref. 1. First we recognize that we are interested in just one term of $\mathbf{S}^{(\phi)}$ $=\Sigma_{\alpha} \mathbf{S}^{(\phi) \alpha}$ in Eq. (7.14). In the expansion of $\mathbf{S}^{(\phi) \alpha}$ the first term is zero by Newton's third law, and the second term is $-\left[\boldsymbol{\nabla} \cdot \boldsymbol{\pi}^{(\phi) \alpha}\right]$, in which $\boldsymbol{\pi}^{(\phi) \alpha}$ is just the contribution of species $\alpha$ to the intramolecular contribution to the stress tensor $\boldsymbol{\pi}^{(\phi)}=\Sigma_{\alpha} \boldsymbol{\pi}^{(\phi) \alpha}$.

(ii) The third term, that containing the external force $\mathbf{F}_{\nu}^{(e) \alpha}$, can be developed similarly to the method for going from Eq. (7.10) to Eqs. (7.11) and (7.12) in Ref. 1. We are interested in just one term in $\mathbf{S}^{(e)}=\Sigma_{\alpha} \mathbf{S}^{(e) \alpha}$. In doing the expansion we get $\mathbf{S}^{(e) \alpha}=\mathbf{G}_{\alpha}-\left[\boldsymbol{\nabla} \cdot \boldsymbol{\pi}^{(e) \alpha}\right]$. Here $\mathbf{G}_{\alpha}$ is the force per unit volume on species $\alpha$, and $\boldsymbol{\pi}^{(e) \alpha}$ is the contribution of species $\alpha$ to the external force contribution to the stress tensor $\pi^{(e)}=\Sigma_{\alpha} \pi^{(e) \alpha}$.

(iii) The second term requires special attention. First we introduce $\mathbf{F}_{v}^{(d) \alpha}$ from Eq. (2.3) into the second term in Eq. (3.6), which gives

$$
\begin{aligned}
-\sum_{\nu \beta \mu} & \iint \delta\left(\mathbf{r}_{\nu}^{\alpha}-\mathbf{r}\right) \mathbf{F}_{\nu \mu}^{(d) \alpha \beta} g_{\alpha \beta, 0}\left(\mathbf{r}^{\alpha}, \mathbf{r}^{\beta}\right) f_{\beta}\left(x^{\beta}, t\right) \\
& \times \Psi_{\alpha} d \mathbf{r}^{\alpha} d x^{\beta} \\
= & -\sum_{\nu \beta \mu} \iint \delta\left(\mathbf{r}_{\nu}^{\alpha}-\mathbf{r}\right) \mathbf{F}_{\nu \mu}^{(d) \alpha \beta} g_{\alpha \beta, 0}\left(\mathbf{r}^{\alpha}, \mathbf{r}^{\beta}\right) \Psi_{\alpha}\left(\mathbf{r}^{\alpha}, t\right) \\
& \times \Psi_{\beta}\left(\mathbf{r}^{\beta}, t\right) d \mathbf{r}^{\alpha} d \mathbf{r}^{\beta} .
\end{aligned}
$$

The triple product $g_{\alpha \beta} \Psi_{\alpha} \Psi_{\beta}$, where each factor is a nonequilibrium property is equivalent to the function $\Psi_{\alpha \beta}$ appearing in Eq. (7.17) of Ref. 1. Here, however, we have the product $\widetilde{\Psi}_{\alpha \beta}^{0}=g_{\alpha \beta, 0} \Psi_{\alpha} \Psi_{\beta}$, containing a local equilibrium radial distribution function. This difference arises because of the restriction in the development of Ref. 3 to the singlemolecule distribution function as discussed on p. 9916 in the final paragraph of the concluding section.

We can then change the variables of integration and rewrite the last equation as

$$
\begin{aligned}
& -\sum_{\nu \beta \mu} \iiint \int \delta\left(\mathbf{r}_{\nu}^{\alpha}-\mathbf{r}\right) \mathbf{F}_{\nu \mu}^{(d) \alpha \beta} \\
& \quad \times \widetilde{\Psi}_{\alpha \beta}^{0}\left(\mathbf{r}_{c}^{\alpha \beta}, \mathbf{R}_{\alpha \beta}, Q^{\alpha}, Q^{\beta}, t\right) d \mathbf{r}_{c}^{\alpha \beta} d \mathbf{R}_{\alpha \beta} d Q^{\alpha} d Q^{\beta} \\
& \quad=-\mathbf{F}^{(d) \alpha}+\left[\nabla \cdot \tilde{\boldsymbol{\pi}}^{(d) \alpha}\right]
\end{aligned}
$$

in which $\mathbf{r}_{\nu}^{\alpha}=\mathbf{r}_{c}^{\alpha \beta}-\left[m^{\beta} /\left(m^{\alpha}+m^{\beta}\right)\right] \mathbf{R}_{\alpha \beta}+\mathbf{R}_{\nu}^{\alpha}$, where $m^{\alpha}$ and $m^{\beta}$ are the total masses of the molecules. The second line of Eq. (3.8) is obtained by following the transformation in going from Eq. (7.16) to (7.17) of Ref. 1. The tensor $\tilde{\pi}^{(d) \alpha}$ is one term (for a particular value of $\alpha$ ) in the sum on the 
right-hand side of Eq. (7.17) (except for the use of $\widetilde{\Psi}_{\alpha \beta}^{0}$ rather than $\left.\widetilde{\Psi}_{\alpha \beta}\right)$. Furthermore we note that $\Sigma_{\alpha} \tilde{\pi}^{(d) \alpha}$ $=\boldsymbol{\pi}^{(d)}$, where $\boldsymbol{\pi}^{(d)}$ is given in Eq. (7.17).

The vector $\mathbf{F}^{(d) \alpha}$ is the analog of $\mathbf{G}_{\alpha}$, namely

$$
\begin{aligned}
\mathbf{F}^{(d) \alpha}= & \sum_{\nu \beta \mu} \iiint \mathbf{F}_{\nu \mu}^{(d) \alpha \beta} \widetilde{\Psi}_{\alpha \beta}^{0}\left(\mathbf{r}, \mathbf{R}_{\alpha \beta}, Q^{\alpha}, Q^{\beta}, t\right) \\
& \times d \mathbf{R}_{\alpha \beta} d Q^{\alpha} d Q^{\beta} .
\end{aligned}
$$

Furthermore the same arguments regarding Newton's third law given in Ref. 1 enable us to show that $\Sigma_{\alpha} \mathbf{F}^{(d) \alpha}=0$. The quantity $\mathbf{F}^{(d) \alpha}$ may be interpreted as the force per unit volume exerted on a molecule of species $\alpha$ by all the other molecules. The distribution function $\widetilde{\Psi}_{\alpha \beta}^{0}$ may be considered to be the sum of a local equilibrium term $\widetilde{\Psi}_{\alpha \beta, 0}^{0}$ and a term arising from the gradients of the macroscopic variables.

For an isotropic system, the local equilibrium term $\widetilde{\Psi}_{\alpha \beta, 0}^{0}$ is isotropic, that is, invariant to a rotation of the coordinate system. Therefore this term does not contribute to the integral in Eq. (3.9). Thus $\mathbf{F}^{(d) \alpha}$ involves only the nonequilibrium contribution to $\widetilde{\Psi}_{\alpha \beta}^{0}$ and therefore must be of the form $\left[\boldsymbol{\nabla} \cdot\left(\boldsymbol{\pi}^{(d) \alpha}-\tilde{\boldsymbol{\pi}}^{(d) \alpha}\right)\right]$, where the difference in the parentheses is a tensor. We can then write the term in Eq. (3.8) as simply $\left[\nabla \cdot \boldsymbol{\pi}^{(d) \alpha}\right]$ (except for the use of $\widetilde{\Psi}_{\alpha \beta}^{0}$ rather than $\widetilde{\Psi}_{\alpha \beta}$ ), and then we have in addition $\Sigma_{\alpha} \pi^{(d) \alpha}=\pi^{(d)}$ since $\Sigma_{\alpha} \mathbf{F}^{(d) \alpha}=0$.

To summarize, Eq. (3.6) becomes

$$
3 \mathrm{rd}=\left[\boldsymbol{\nabla} \cdot \boldsymbol{\pi}^{(\phi) \alpha}\right]+\left[\boldsymbol{\nabla} \cdot \boldsymbol{\pi}^{(d) \alpha}\right]+\left[-\mathbf{G}_{\alpha}+\boldsymbol{\nabla} \cdot \boldsymbol{\pi}^{(e) \alpha}\right] .
$$

After combining the three terms [Eqs. (3.4), (3.5), and (3.6)] and performing some regrouping of the terms, the left-hand side of Eq. (3.2) becomes finally

$$
\begin{aligned}
\text { Left-hand side }= & \mathbf{v}\left(\frac{\partial \rho_{\alpha}}{\partial t}+\boldsymbol{\nabla} \cdot \rho_{\alpha} \mathbf{v}\right)+\rho_{\alpha}\left[\frac{\partial \mathbf{v}}{\partial t}+\mathbf{v} \cdot \boldsymbol{\nabla} \mathbf{v}\right] \\
& +\left[\boldsymbol{\nabla} \cdot \boldsymbol{\pi}_{\alpha}\right]-\mathbf{G}_{\alpha}
\end{aligned}
$$

in which $\boldsymbol{\pi}_{\alpha}=\boldsymbol{\pi}^{(k) \alpha}+\boldsymbol{\pi}^{(\phi) \alpha}+\boldsymbol{\pi}^{(e) \alpha}+\boldsymbol{\pi}^{(d) \alpha}$ is the contribution of species $\alpha$ to the stress tensor. According to Ref. 1, Eq. (6.1), the quantity in the first set of parentheses equals $-\left(\boldsymbol{\nabla} \cdot \mathbf{j}_{\alpha}\right)$. But the latter, when evaluated using Ref. 1, Eq. (6.10), line 1 , is seen to be zero when $f_{\alpha 0}$ is used for the singlet phase space distribution. The quantity in the second set of parentheses is, according to Eq. (7.1) of Ref. 1, $(1 / \rho)[-\nabla \cdot \boldsymbol{\pi}+\mathbf{G}]$, in which $\boldsymbol{\pi}=\boldsymbol{\pi}^{(k)}+\boldsymbol{\pi}^{(\phi)}+\boldsymbol{\pi}^{(e)}+\boldsymbol{\pi}^{(d)}$. Therefore Eq. (3.11) becomes

$$
\text { Left-hand side }=\frac{\rho_{\alpha}}{\rho}[-\nabla \cdot \boldsymbol{\pi}+\mathbf{G}]+\left[\boldsymbol{\nabla} \cdot \boldsymbol{\pi}_{\alpha}-\mathbf{G}_{\alpha}\right] \equiv \mathbf{d}_{\alpha} .
$$

The symbol $\mathbf{d}_{\alpha}$, introduced here is a generalization of the $\mathbf{d}_{\alpha}$ in Eq. (11.2-28) of Ref. 9. The latter is the generalized driving force for diffusion of substance $\alpha$ in the linear limit of irreversible thermodynamics. Note that for the $\mathbf{d}_{\alpha}$ defined here, we also have $\Sigma_{\alpha} \mathbf{d}_{\alpha}=0$.

\section{B. Right-hand side of Eq. (3.2)}

We now consider some assumptions for $\mathbf{E}_{\nu \mu}^{\alpha \beta}$ that lead to the Maxwell-Stefan equations. We begin by assuming that the self-correlation coefficients are nonzero only when the beads on two different molecules coincide (the "selfcorrelation localization assumption")

$$
\mathbf{E}_{\nu \mu}^{\alpha \beta}=\overline{\mathbf{E}}_{\nu \mu}^{\alpha \beta} \delta\left(\mathbf{r}_{\nu}^{\alpha}-\mathbf{r}_{\mu}^{\beta}\right) .
$$

This assumption does not seem to be as stringent as the "short-range force assumption" 1 introduced earlier and is discussed further in connection with Eq. (4.14). Then,

$\begin{aligned} \text { Right-hand side }= & \sum_{\beta \mu \nu} \frac{1}{\sqrt{k T}} h_{\alpha}^{[0]} h_{\beta}^{[0]} \iint \delta\left(\mathbf{r}_{\nu}^{\alpha}-\mathbf{r}\right) \\ & \times e^{-P^{\alpha 2}} e^{-P^{\beta 2}} \overline{\mathbf{E}}_{\nu \mu}^{\alpha \beta} \delta\left(\mathbf{r}_{\nu}^{\alpha}-\mathbf{r}_{\mu}^{\beta}\right) \\ & \cdot\left[\frac{1}{\sqrt{m_{\mu}^{\beta}}} \mathbf{f}_{\beta \mu}^{[1]} f_{\alpha}^{[0]}-\frac{1}{\sqrt{m_{\nu}^{\alpha}}} \mathbf{f}_{\alpha \nu}^{[1]} f_{\beta}^{[0]}\right] d x^{\alpha} d x^{\beta} .\end{aligned}$

Clearly, in this expression, $\delta\left(\mathbf{r}_{\nu}^{\alpha}-\mathbf{r}_{\mu}^{\beta}\right)$ can be replaced by $\delta\left(\mathbf{r}_{\mu}^{\beta}-\mathbf{r}\right)$. Next, from Ref. 3, Appendix A:

$$
\begin{aligned}
& f_{\alpha}^{[0]}=\frac{h_{\alpha}^{[0]}}{J_{\alpha}} \Psi_{\alpha}, \\
& \mathbf{f}_{\alpha \nu}^{[1]}=\frac{h_{\alpha}^{[0]}}{J_{\alpha}} \sqrt{\frac{m_{\nu}^{\alpha}}{k T}}\left[\left[\dot{\mathbf{r}}_{\nu}^{\alpha}-\mathbf{v}(\mathbf{r}, t)\right]\right]^{\alpha} \Psi_{\alpha} .
\end{aligned}
$$

The notation for momentum-space averages $[[\cdots]]^{\alpha}$ is explained in Sec. 5.1 of Ref. 1. We now define a momentum average of the self-correlation tensor as

$\left(h_{\alpha}^{[0]} h_{\beta}^{[0]}\right)^{2} \iint e^{-P^{\alpha 2}} e^{-P^{\beta 2}} \overline{\mathbf{E}}_{\nu \mu}^{\alpha \beta} d \mathbf{P}^{\alpha} d \mathbf{P}^{\beta}=\left[\left[\overline{\mathbf{E}}_{\nu \mu}^{\alpha \beta}\right]\right]_{0}$,

where the subscript " 0 ", indicates that the average is performed using the equilibrium distribution. Then we get

$$
\begin{aligned}
\text { Right-hand side }= & \sum_{\beta \nu \mu} \frac{1}{k T} \iint \delta\left(\mathbf{r}_{\nu}^{\alpha}-\mathbf{r}\right) \delta\left(\mathbf{r}_{\mu}^{\beta}-\mathbf{r}\right) \\
& \times \Psi_{\alpha} \Psi_{\beta}\left[\left[\overline{\mathbf{E}}_{\nu \mu}^{\alpha \beta}\right]\right]_{0} \cdot\left(\left[\left[\dot{\mathbf{r}}_{\mu}^{\beta}-\mathbf{v}(\mathbf{r}, t)\right]\right]^{\beta}\right. \\
& \left.-\left[\left[\dot{\mathbf{r}}_{\nu}^{\alpha}-\mathbf{v}(\mathbf{r}, t)\right]\right]^{\alpha}\right) d \mathbf{r}^{\alpha} d \mathbf{r}^{\beta} .
\end{aligned}
$$

Note that if the $\overline{\mathbf{E}}_{\nu \mu}^{\alpha \beta}$ do not depend on momenta, then $\left[\left[\overline{\mathbf{E}}_{\nu \mu}^{\alpha \beta}\right]\right]_{0}=\overline{\mathbf{E}}_{\nu \mu}^{\alpha \beta}$.

If we further define a set of symmetric second-order friction tensors $\mathbf{Z}_{\nu \mu}^{\alpha \beta}$ by

$m_{\nu}^{\alpha} \int \delta\left(\mathbf{r}_{\nu}^{\alpha}-\mathbf{r}\right) \Psi_{\alpha}\left[\left[\overline{\mathbf{E}}_{\nu \mu}^{\alpha \beta}\right]\right]_{0} d \mathbf{r}^{\alpha}=\rho_{\nu}^{\alpha}(\mathbf{r}) \mathbf{Z}_{\nu \mu}^{\alpha \beta}\left(\mathbf{r}, \mathbf{r}^{\beta}, t\right)$

(where $\rho_{\nu}^{\alpha}$ is the mass density of beads), then 
Right-hand side $=\sum_{\beta \nu \mu} \frac{1}{k T}\left(\frac{1}{m_{\nu}^{\alpha}} \int \rho_{\nu}^{\alpha}(\mathbf{r}) \delta\left(\mathbf{r}_{\mu}^{\beta}-\mathbf{r}\right) \Psi_{\beta} \mathbf{Z}_{\nu \mu}^{\alpha \beta}\right.$

$$
\begin{aligned}
& \cdot\left[\left[\dot{\mathbf{r}}_{\mu}^{\beta}-\mathbf{v}(\mathbf{r}, t)\right]\right]^{\beta} d \mathbf{r}^{\beta}-\frac{1}{m_{\mu}^{\beta}} \int \rho_{\mu}^{\beta}(\mathbf{r}) \\
& \times \delta\left(\mathbf{r}_{\nu}^{\alpha}-\mathbf{r}\right) \Psi_{\alpha} \mathbf{Z}_{\mu \nu}^{\beta \alpha} \\
& \left.\cdot\left[\left[\dot{\mathbf{r}}_{\nu}^{\alpha}-\mathbf{v}(\mathbf{r}, t)\right]\right]^{\alpha} d \mathbf{r}^{\alpha}\right) .
\end{aligned}
$$

Note that if $\left[\left[\overline{\mathbf{E}}_{\nu \mu}^{\alpha \beta}\right]\right]_{0}$ is independent of all the configuration coordinates $\mathbf{r}_{\nu}^{\alpha}$, then $\mathbf{Z}_{\nu \mu}^{\alpha \beta}=\left[\left[\overline{\mathbf{E}}_{\nu \mu}^{\alpha \beta}\right]\right]_{0}$.

The first integral in Eq. (3.20) may be written as

$\left[\frac{1}{m_{\nu}^{\alpha} m_{\mu}^{\beta}} \rho_{\nu}^{\alpha}(\mathbf{r})\right] m_{\mu}^{\beta} \int \delta\left(\mathbf{r}_{\mu}^{\beta}-\mathbf{r}\right) \Psi_{\beta} \mathbf{Z}_{\nu \mu}^{\alpha \beta} \cdot\left[\left[\dot{\mathbf{r}}_{\mu}^{\beta}-\mathbf{v}(\mathbf{r}, t)\right]\right]^{\beta} d \mathbf{r}^{\beta}$.

However, according to line 1 of Eq. (6.10) of Ref. 1, the integral in Eq. (3.21) is closely related to the mass flux:

$$
\mathbf{j}_{\beta}=\sum_{\mu} \mathbf{j}_{\mu}^{\beta}=\sum_{\mu} m_{\mu}^{\beta} \int \delta\left(\mathbf{r}_{\mu}^{\beta}-\mathbf{r}\right) \Psi_{\beta}\left[\left[\dot{\mathbf{r}}_{\mu}^{\beta}-\mathbf{v}(\mathbf{r}, t)\right]\right]^{\beta} d \mathbf{r}^{\beta} .
$$

Here $\mathbf{j}_{\mu}^{\beta}$ is the mass flux of beads $\mu$ of species $\beta$, and $\rho_{\mu}^{\beta}$ is the corresponding density. Hence this suggests defining a weighted average of $\mathbf{Z}_{\nu \mu}^{\alpha \beta}$ thus

$$
m_{\mu}^{\beta} \int \delta\left(\mathbf{r}_{\mu}^{\beta}-\mathbf{r}\right) \Psi_{\beta} \mathbf{Z}_{\nu \mu}^{\alpha \beta} \cdot\left[\left[\dot{\mathbf{r}}_{\mu}^{\beta}-\mathbf{v}(\mathbf{r}, t)\right]\right]^{\beta} d \mathbf{r}^{\beta}=\overline{\mathbf{Z}}_{\nu \mu}^{\alpha \beta} \cdot \mathbf{j}_{\mu}^{\beta} .
$$

If $\mathbf{Z}_{\nu \mu}^{\alpha \beta}$ is independent of $\mathbf{r}^{\beta}$, then $\overline{\mathbf{Z}}_{\nu \mu}^{\alpha \beta}=\mathbf{Z}_{\nu \mu}^{\alpha \beta}$. Hence

$$
\text { Right-hand side }=\sum_{\beta \nu \mu} \frac{\rho_{\nu}^{\alpha} \rho_{\mu}^{\beta}}{m_{\nu}^{\alpha} m_{\mu}^{\beta} k T}\left[\overline{\mathbf{Z}}_{\nu \mu}^{\alpha \beta} \cdot \frac{\mathbf{j}_{\mu}^{\beta}}{\rho_{\mu}^{\beta}}-\overline{\mathbf{Z}}_{\mu \nu}^{\beta \alpha} \cdot \frac{\mathbf{j}_{\nu}^{\alpha}}{\rho_{\nu}^{\alpha}}\right] .
$$

Now we take the $\overline{\mathbf{Z}}_{\nu \mu}^{\alpha \beta}$ to be independent of the bead number so that $\overline{\mathbf{Z}}_{\nu \mu}^{\alpha \beta}=\overline{\mathbf{Z}}_{\alpha \beta}$, and assume that the beads in each type of molecule are all identical so that $m_{\nu}^{\alpha}=m_{0}^{\alpha}$ for all $\nu$. Then since $\overline{\mathbf{Z}}_{\alpha \beta}$ is symmetric with respect to the interchange of indices $\alpha$ and $\beta$

$$
\text { Right-hand side }=\sum_{\beta} \frac{\overline{\mathbf{Z}}_{\alpha \beta} \rho_{\alpha} \rho_{\beta}}{m_{0}^{\alpha} m_{0}^{\beta} k T} \cdot\left[\frac{\mathbf{j}_{\beta}}{\rho_{\beta}}-\frac{\mathbf{j}_{\alpha}}{\rho_{\alpha}}\right] .
$$

When the expressions in Eqs. (3.12) and (3.25) are equated, we get Eq. (1.5), which is the principal result of this paper.

\section{CONNECTION WITH THE RESULTS FOR A DILUTE GAS OF RIGID SPHERES}

The above development has been presented as a kinetic theory of polymers. However, by allowing the bead labels $\nu$ and $\mu$ to assume only the value of " 1 ", we can also regard this as a theory for monatomic liquids and gases. In this section we show that the present theory gives exactly the same result for the diffusivity of a dilute gas composed of rigid spheres as is obtained from the Chapman-Enskog theory. This confirms that the development of the FokkerPlanck equation in Ref. 3 and here in Secs. II and III is consistent with the Boltzmann equation and the ChapmanEnskog solution thereof.

\section{A. Results from the Chapman-Enskog theory}

If $\overline{\mathbf{Z}}_{\alpha \beta}$ is taken to be isotropic, so that $\overline{\mathbf{Z}}_{\alpha \beta}=\bar{Z}_{\alpha \beta} \boldsymbol{\delta}$, where $\delta$ is the unit tensor, we then get

$\sum_{\beta} \frac{\bar{Z}_{\alpha \beta} \rho_{\alpha} \rho_{\beta}}{m_{0}^{\alpha} m_{0}^{\beta} k T}\left[\frac{\mathbf{j}_{\alpha}}{\rho_{\alpha}}-\frac{\mathbf{j}_{\beta}}{\rho_{\beta}}\right]=-\left[\boldsymbol{\nabla} \cdot \boldsymbol{\pi}_{\alpha}-\mathbf{G}_{\alpha}\right]+\frac{\rho_{\alpha}}{\rho}[\boldsymbol{\nabla} \cdot \boldsymbol{\pi}-\mathbf{G}]$.

We note that this result differs from that of the irreversible thermodynamics in Eq. (1.4) in that there are no terms describing thermal diffusion and that Eq. (4.1) contains terms with the complete stress tensor, which for dilute gases simplify to pressure and partial pressure terms in Eq. (1.4). Comparison of the two results enables us to get the correspondence between the $\widetilde{C}_{\alpha \beta}$ of Eq. (1.4) and the $\bar{Z}_{\alpha \beta}$ of Eq. (4.1):

$$
c R T \widetilde{C}_{\alpha \beta}=\frac{\rho_{\alpha} \rho_{\beta}}{m_{0}^{\alpha} m_{0}^{\beta} k T} \bar{Z}_{\alpha \beta} .
$$

We further know that, for a dilute gas, to a very good approximation $^{4}$ (probably within $3 \%$ )

$$
\widetilde{C}_{\alpha \beta}=\frac{x_{\alpha} x_{\beta}}{\mathcal{D}_{\alpha \beta}},
$$

where the $x_{\alpha}$ are mole fractions, and the $\mathcal{D}_{\alpha \beta}$ are binary diffusivities.

From Eq. (8.2-9) of Ref. 9, or Eq. (14.2-1) of Ref. 10, it is known that for dilute binary gas mixtures of rigid spheres

$$
\mathcal{D}_{\alpha \beta}=\frac{3}{16 n \pi \sigma^{2}} \sqrt{\frac{2 \pi k T}{m_{\alpha \beta}}} \text {. }
$$

Here $n$ is the number density of the binary gas mixture, $\sigma$ $=\frac{1}{2}\left(\sigma_{\alpha}+\sigma_{\beta}\right)$ is the collision diameter for the binary pair, and $m_{\alpha \beta}$ is the reduced mass of the binary pair (the harmonic mean of the individual masses).

Combination of the three formulas just given then gives the "friction coefficient" $\bar{Z}_{\alpha \beta}$ in terms of the molecular parameters as

$$
\bar{Z}_{\alpha \beta}=\frac{(k T)^{2}}{n \mathcal{D}_{\alpha \beta}}=\frac{16 \pi \sigma^{2}(k T)^{2}}{3} \sqrt{\frac{m_{\alpha \beta}}{2 \pi k T}} .
$$

This suggests that, for ideal gas mixtures, the friction coefficient is approximately proportional to the $3 / 2$ power of the temperature.

\section{B. Results from simplifying the polymer theory}

Next we want to show that the same result is obtained from the expression for the self-correlation tensors, for the limiting case of a dilute gas of rigid spheres. The selfcorrelation tensors are defined by Eq. (6.7) of Ref. 3. In transforming this equation into Eq. (7.7), we take the time 
interval $\tau$ to be long compared with the duration of a collision, but short compared with the time interval between collisions. This leads to

$$
\begin{aligned}
\mathbf{E}_{\nu \mu}^{\alpha i, \beta j} & =\int_{0}^{\tau} \mathbf{F}_{\nu \mu}^{(d) \alpha i, \beta j} e^{-s \mathcal{L}} \mathbf{F}_{\nu \mu}^{(d) \alpha i, \beta j} d s \\
& =\mathbf{F}_{\nu \mu}^{(d) \alpha i, \beta j} \int_{0}^{\tau} e^{-s \mathcal{L}} \mathbf{F}_{\nu \mu}^{(d) \alpha i, \beta j} d s
\end{aligned}
$$

with the Liouville operator $\mathscr{L}$ being the operator associated with a pair of colliding atoms. We retain the indices $\nu$ and $\mu$ even though it is understood that in this section both are "1.",

We let $m_{\alpha \beta}$ be the reduced mass of a pair of colliding atoms $m_{\alpha \beta}=m_{\nu}^{\alpha} m_{\mu}^{\beta} /\left(m_{\nu}^{\alpha}+m_{\mu}^{\beta}\right)$ and their relative velocities be $\mathbf{g}=\mathbf{v}_{\nu}^{\alpha i}-\mathbf{v}_{\mu}^{\beta j}$, so that for dilute monatomic gases (i.e., during the time interval during which the motions of beads $\alpha i \nu$ and $\beta j \mu$ are not affected by a third bead in the immediate neighborhood)

$$
\mathbf{F}_{\nu \mu}^{(d) \alpha i, \beta j}=m_{\alpha \beta} \frac{d}{d t} \mathbf{g}
$$

and

$$
\int_{0}^{\tau} e^{-s \mathcal{L}} \mathbf{F}_{\nu \mu}^{(d) \alpha i, \beta j} d s=m_{\alpha \beta}\left(\mathbf{g}-\mathbf{g}^{(0)}\right),
$$

where $\mathbf{g}^{(0)}$ is the "initial relative velocity," that is, the relative velocity at the beginning of a collision (i.e., at the beginning of the time interval under consideration). Let $\mathbf{k}$ be a unit vector between the two colliding rigid spheres at the point of closest approach and fix the sign by requiring that $(\mathbf{g} \cdot \mathbf{k})>0$. Then it may be shown (Ref. 10, p. 57) that $\mathbf{g}$ $-\mathbf{g}^{(0)}=2[\mathbf{k k} \cdot \mathbf{g}]$ so that

$$
\int_{0}^{\tau} e^{-s \mathcal{L}} \mathbf{F}_{\nu \mu}^{(d) \alpha i, \beta j} d s=2 m_{\alpha \beta}[\mathbf{k k} \cdot \mathbf{g}] .
$$

However, $\mathbf{F}_{\nu \mu}^{(d) \alpha i, \beta j}$ also may be written as $-(\partial / \partial \mathbf{R}) \varphi$, where $\varphi$ is the interbead potential energy, and $\mathbf{R}$ is the interbead vector (between bead $\alpha i \nu$ and bead $\beta j \mu$ ). Therefore

$$
\mathbf{E}_{\nu \mu}^{\alpha i, \beta j}=-\left(\frac{\partial}{\partial \mathbf{R}} \varphi\right) 2 m_{\alpha \beta}[\mathbf{k k} \cdot \mathbf{g}] .
$$

Then from Eq. (7.8) in Ref. 3

$$
\begin{aligned}
\mathbf{E}_{\nu \mu}^{\alpha \beta} f_{\alpha 0} f_{\beta 0}= & \sum_{i j} \int \mathbf{E}_{\nu \mu}^{\alpha i, \beta j} \delta\left(x^{\alpha i}-x^{\alpha}\right) \delta\left(x^{\beta j}-x^{\beta}\right) \\
& \times f_{h 0}^{\alpha \beta}(x, t) d x
\end{aligned}
$$

in which $f_{h 0}^{\alpha \beta}(x, t)$ is the distribution function equilibrated in momentum space. In the integration over all $x$, in a dilute gas, at any one time there will be at most one interaction, which is taken to be between beads $\alpha i \nu$ and $\beta j \mu$. This means that Eq. (4.11) may be reduced to

$$
\begin{aligned}
\mathbf{E}_{\nu \mu}^{\alpha \beta} f_{\alpha 0} f_{\beta 0}= & \sum_{i j} \int \mathbf{E}_{\nu \mu}^{\alpha i, \beta j}\left(x_{\nu}^{\alpha i}, x_{\mu}^{\beta j}\right) \delta\left(x^{\alpha i}-x^{\alpha}\right) \\
& \times \delta\left(x^{\beta j}-x^{\beta}\right) f_{h 0}^{\alpha \beta}\left(x^{\alpha i}, x^{\beta j}, t\right) d x^{\alpha} d x^{\beta} \\
= & \mathbf{E}_{\nu \mu}^{\alpha i, \beta j} f_{\alpha 0} f_{\beta 0} g_{\alpha \beta, 0}
\end{aligned}
$$

in which, in the first form, $f_{h 0}^{\alpha \beta}\left(x^{\alpha i}, x^{\beta j}, t\right)$ is understood to be $f_{h 0}^{\alpha \beta}(x, t)$ integrated over all $x$ except $x^{\alpha i}$ and $x^{\beta j}$ [see Eq. (6.5) of Ref 3].

Then using Eq. (4.10) we get

$$
\begin{aligned}
\mathbf{E}_{\nu \mu}^{\alpha \beta} & =-2 m_{\alpha \beta} e^{-\varphi / k T}\left(\frac{\partial}{\partial \mathbf{R}} \varphi\right)[\mathbf{k k} \cdot \mathbf{g}] \\
& =2 m_{\alpha \beta} k T\left(\frac{\partial}{\partial \mathbf{R}}\left(e^{-\varphi / k T}-1\right)\right)[\mathbf{k k} \cdot \mathbf{g}] .
\end{aligned}
$$

In the limit that the potential $\varphi(R)$ is that of a rigid sphere, $\exp (-\varphi / k T)$ is zero inside a sphere of radius $\sigma$, and also zero outside. Then the factor $(\partial / \partial \mathbf{R})(\cdots)$ is zero throughout the space of $\mathbf{R}$, except on the surface of radius $\sigma$, on which it is a vector in the direction of $\mathbf{k}$. That is, $\mathbf{E}_{\nu \mu}^{\alpha \beta}$ will be some multiple of the Dirac delta function:

$$
\mathbf{E}_{\nu \mu}^{\alpha \beta}=C \delta(\mathbf{R}-\sigma \mathbf{k}) .
$$

To determine the multiplicative constant $C$ we integrate both sides of Eq. (4.14) over all $\mathbf{R}$ for which $(\mathbf{k} \cdot \mathbf{g})>0$. Using Eq. (4.13) this gives

$$
\begin{aligned}
C & =2 m_{\alpha \beta} k T \iint\left(\frac{\partial}{\partial R}\left(e^{-\varphi / k T}-1\right)\right) \mathbf{k}[\mathbf{k} \mathbf{k} \cdot \mathbf{g}] R^{2} d R d \mathbf{k} \\
& =2 m_{\alpha \beta} k T \sigma^{2} \int \mathbf{k} \mathbf{k}(\mathbf{k} \cdot \mathbf{g}) d \mathbf{k} \\
& =\frac{2}{3} m_{\alpha \beta} k T \sigma^{2} \boldsymbol{\delta} \int(\mathbf{k} \cdot \mathbf{g}) d \mathbf{k}=\frac{4}{3} \pi m_{\alpha \beta} k T \sigma^{2} \boldsymbol{\delta} g
\end{aligned}
$$

in which $g$ is the magnitude of $\mathbf{g}$. Thus we have found

$$
\mathbf{E}_{\nu \mu}^{\alpha \beta}=\frac{4}{3} \pi m_{\alpha \beta} k T \sigma^{2} g \boldsymbol{\delta} \delta(\mathbf{R}-\sigma \mathbf{k})
$$

for the self-correlation tensors. This result is to be compared with the assumption we made in Eq. (3.13), which, in the present notation, is $\mathbf{E}_{\nu \mu}^{\alpha \beta}=\overline{\mathbf{E}}_{\nu \mu}^{\alpha \beta} \delta(\mathbf{R})$. For dilute gases, where the size of a molecule is small compared with the mean free path, the neglect of the $\sigma \mathbf{k}$ in the argument of the delta function is not serious; however, inclusion of the $\sigma \mathbf{k}$ would lead to the Enskog dense gas theory.

Finally we get the momentum averaged quantity $\left[\left[\overline{\mathbf{E}}_{\nu \mu}^{\alpha \beta}\right]\right]_{0}$ :

$$
\begin{aligned}
{\left[\left[\overline{\mathbf{E}}_{\nu \mu}^{\alpha \beta}\right]\right]_{0} } & =\frac{4}{3} \pi m_{\alpha \beta} k T \sigma^{2} \boldsymbol{\delta} \frac{\iint e^{-P^{\alpha 2}} e^{-P^{\beta 2}} g d P^{\alpha} d P^{\beta}}{\iint e^{-P^{\alpha 2}} e^{-P^{\beta 2}} d P^{\alpha} d P^{\beta}} \\
& =\frac{4}{3} \pi m_{\alpha \beta} k T \sigma^{2} \boldsymbol{\delta} \frac{\int e^{-m_{\alpha \beta} g^{2} / 2 k T} g d \mathbf{g}}{\int e^{-m_{\alpha \beta} g^{2} / 2 k T} d \mathbf{g}} \\
& =\frac{4}{3} \pi m_{\alpha \beta} k T \sigma^{2} \sqrt{\frac{8 k T}{\pi m_{\alpha \beta}}} \boldsymbol{\delta} \\
& =\frac{16}{3} \pi \sigma^{2}(k T)^{2} \sqrt{\frac{m_{\alpha \beta}}{2 \pi k T}} \boldsymbol{\delta}
\end{aligned}
$$

which is in exact agreement with the result given in Eq. (4.5), since $\bar{Z}_{\alpha \beta}=\left[\left[\overline{\mathbf{E}}_{\nu \mu}^{\alpha \beta}\right]\right]_{0}$ for a system composed of atoms. 
In this section we have shown that we get the same expression for the friction coefficients from the ChapmanEnskog kinetic theory and from the correlation function for the special case of a dilute gas of rigid spheres. We have also seen the connection with the Enskog kinetic theory of dense gases [see remarks after Eq. (4.16)].

The next step should be to get the friction tensor for polymeric systems. One could use the bead-spring chain model in dilute solutions, or the bead-spring chain model for concentrated solutions and melts, in which Lennard-Jones type of forces have to be introduced to take care of chainchain interaction. Clearly this will be quite a challenge.

\section{CONCLUDING COMMENTS}

There are three distinct types of assumptions that have been made in this development, and it is important to distinguish among them.

\section{A. The perturbation series in $\varepsilon$}

This series was presented in Ref. 3 in detail, where it is shown that the parameter $\varepsilon$ arises as the ratio of two time constants. This perturbation scheme is analogous to the Chapman-Enskog expansion for gases, in which the first approximation yields the usual transport equations (equation of continuity, the Navier-Stokes equations, and the energy equation) and the second-order approximation (which leads to the Burnett equations). It is interesting to note that, at first order, the Chapman-Enskog theory gives a stress tensor that is a linear relation between stresses and velocity gradients, whereas the lowest level treatment presented here for polymers gives nonlinear relations for the stress tensor (i.e., nonNewtonian effects). The reason for this lies in the inclusion of intermolecular and intramolecular force contributions to the stress tensor through the nonequilibrium configurational distribution function on the right-hand side of Eq. (2.6). As pointed out after Eq. (2.6) this factor is simply the local number density in the treatments of monatomic gases and liquids.

\section{B. The expansion in tensor Hermite polynomials}

In the Chapman-Enskog kinetic theory of gases, the Boltzmann equation is solved by an expansion in Sonine polynomials (see p. 475 of Ref. 9). It was found that for realistic intermolecular forces, only the first term in this expansion is needed (Ref. 9, Chap. 8). Here we use the expansion in tensor Hermite polynomials, which were developed in a previous paper. ${ }^{3}$ The tensor Hermite polynomials are analogous to the Sonine polynomials and are needed for the description of molecules with internal structure. By analogy with the dilute gas, at most a few terms will be needed in this expansion; it is shown in Ref. 3 that, with a small number of assumptions, only one term is needed.

In Ref. 3 we solved the Fokker-Planck equation for a well-specified set of assumptions. It was found that there is no coupling between the contributions corresponding to different values of $n$. In this paper, however, we have made fewer assumptions, and these lead to a coupling between different values of $n$. Since we have in this paper terminated the series in the first set of equations at $n=1$, we are not able to describe the thermal diffusion effect. It is for this reason that we do not get a term in the Maxwell-Stefan equations in Eq. (1.5) containing the temperature gradient.

\section{Assumptions related to the self-correlation tensor}

In Sec. III B of this paper we deal with assumptions that enable us to introduce the mass fluxes. In Eq. (3.13) we introduce the "self-correlation localization assumption" which restricts the correlations to situations in which the relevant beads coincide. Then in Eq. (3.17) we perform a momentum averaging, and in Eq. (3.19) a configurational averaging. In Eq. (3.23) a further weighted average is performed. Little is known about the nature of these averages or how crucial they are in polymer kinetic theory. We know only that for dilute monatomic gases the averaging processes are redundant.

The new feature that appears here in the MaxwellStefan form of the diffusion flux equations is that the mass fluxes are operated on by tensors. This means that even in binary systems, the mass flux may not necessarily be collinear with the diffusional driving force. We are unaware of any experiments that have investigated this kind of behavior.

${ }^{1}$ C. F. Curtiss and R. B. Bird, Adv. Polym. Sci. 125, 1 (1996).

${ }^{2}$ C. F. Curtiss and R. B. Bird, Proc. Natl. Acad. Sci. USA 93, 7440 (1996).

${ }^{3}$ C. F. Curtiss and R. B. Bird, J. Chem. Phys. 106, 9899 (1997).

${ }^{4}$ C. F. Curtiss and R. B. Bird, Ind. Eng. Chem. Res. 38, 2515 (1999).

${ }^{5}$ H. C. Öttinger, Rheol. Acta 31, 14 (1992).

${ }^{6}$ A. N. Beris and V. G. Mavrantzas, J. Rheol. 38, 1235 (1994), Eq. (29).

${ }^{7}$ M. W. Johnson, Jr. (unpublished).

${ }^{8}$ J. G. Kirkwood, J. Chem. Phys. 14, 180 (1948).

${ }^{9}$ J. O. Hirschfelder, C. F. Curtiss, and R. B. Bird, Molecular Theory of Gases and Liquids (Wiley, New York, 1954); corrected printing with notes added 1964.

${ }^{10} \mathrm{~S}$. Chapman and T. G. Cowling, The Mathematical Theory of NonUniform Gases, Third ed., prepared in cooperation with D. Burnett (Cambridge University Press, Cambridge, UK, 1970). 